\title{
Calidad y satisfacción de los servicios escolares de una institución de educación superior mexicana: la visión de los estudiantes
}

\author{
Quality and satisfaction of the school services of a Mexican Higher \\ Education Institution: the vision of the students
}

\section{Qualidade e satisfação dos serviços escolares de uma instituição mexicana de ensino superior: a visão dos alunos}

Roger Manuel Patrón-Cortés

Universidad Autónoma de Campeche, México

rmpatron@uacam.mx https://orcid.org/0000-0003-4553-9803

\section{Resumen}

La presente investigación se realizó en el departamento de Servicios Escolares (SE) de una institución de educación superior ubicada en México y tuvo como objetivo medir la calidad del servicio ofrecido desde la percepción de los estudiantes. Este estudio, por tanto, fue exploratorio y descriptivo; primero se realizó una medición cuantitativa y posteriormente se aplicaron entrevistas cualitativas mediante la técnica de grupos focales. Los resultados indican que el servicio que ofrece el departamento de SE es de adecuada calidad, de acuerdo con la percepción de los estudiantes. Las calificaciones más bajas se presentan en las dimensiones capacidad de respuesta y empatía, ya que prevalece como principal inconveniente la actitud que asume una parte del personal del departamento de SE, caracterizada en ocasiones por la falta de amabilidad, disposición y tiempo de respuesta. Se requiere, por tanto, que los administradores inspiren en estos empleados el significado y la importancia que tiene el servicio a los estudiantes, plasmados en su misión, visión y objetivos. Por eso, se propone que la institución fomente una cultura de sensibilidad del servicio, apoyada en la teoría del comportamiento organizacional, en donde todos los trabajadores sean amigables, corteses, accesibles y expertos. Asimismo, 


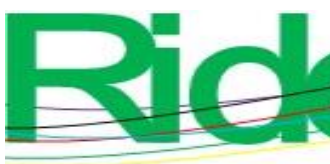

Revista Iberoamericana para la

Investigación y el Desarrollo Educativo

ISSN 2007 - 7467

se recomienda establecer un sistema de evaluación constante en la institución para determinar los niveles de satisfacción en beneficio de la calidad de los servicios escolares ofrecidos.

Palabras clave: calidad del servicio, comportamiento organizacional, satisfacción del usuario.

\section{Abstract}

This study was conducted in the Department of School Services (SE) of a Higher Education Institution located in the southeastern of Mexico and aimed to measure the quality of the service offered from the perception of the students. This study is exploratory and descriptive, first a quantitative measurement was then qualitative interviews were applied through the focus group technique. The results indicate that the service offered by the SE department is of adequate quality according to the perception of the students. The lowest scores are presented in the dimensions: responsiveness and empathy, since the attitude assumed by a part of the SE department staff, characterized by the lack of kindness, willingness and response time, prevails as the main drawback. Administrators are required to inspire in these employees the meaning and importance of service to students, embodied in their mission, vision and objectives. Therefore, it is proposed that the institution foster a culture of service sensitivity, supported by the theory of organizational behavior, where all workers are friendly, courteous, accessible and expert. Likewise, it is recommended to establish a constant evaluation system in the institution, to determine the levels of satisfaction for the benefit of the quality of the school services offered.

Keywords: Quality of service, organizational behavior, user satisfaction.

\section{Resumo}

Esta pesquisa foi realizada no Departamento de Serviços Escolares (SE) de uma instituição de ensino superior localizada no México e teve como objetivo medir a qualidade do serviço oferecido a partir da percepção dos alunos. Este estudo, portanto, foi exploratório e descritivo; primeiramente, foi realizada uma mensuração quantitativa e, posteriormente, foram aplicadas entrevistas qualitativas por meio da técnica de grupo focal. Os resultados indicam que o serviço oferecido pela secretaria de SE é de qualidade adequada, na percepção dos alunos. As pontuações mais baixas são apresentadas nas dimensões de responsividade e empatia, visto que a principal desvantagem é a atitude 


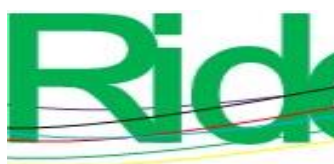

Revista Iberoamericana para la Investigación y el Desarrollo Educativo ISSN $2007-7467$

assumida por parte do pessoal da área de SE, por vezes caracterizada por falta de gentileza, disposição e tempo de resposta. Os administradores devem, portanto, inspirar nesses funcionários o significado e a importância do serviço aos alunos, incorporado em sua missão, visão e objetivos. Portanto, propõe-se que a instituição promova uma cultura de sensibilidade ao serviço, apoiada na teoria do comportamento organizacional, onde todos os trabalhadores sejam amigáveis, corteses, acessíveis e experientes. Da mesma forma, recomenda-se estabelecer um sistema de avaliação constante na instituição para determinar os níveis de satisfação em benefício da qualidade dos serviços escolares oferecidos.

Palavras-chave: qualidade do serviço, comportamento organizacional, satisfação do usuário.

Fecha Recepción: Agosto 2020

Fecha Aceptación: Enero 2021

\section{Introducción}

\section{Concepto de servicio}

En sus inicios, el concepto servicio era considerado como un proceso que servía para añadir valor a un producto, lo cual establecía una relación más estrecha entre el productor y el consumidor. Sin embargo, a partir de la década de los años 80, las definiciones de esta palabra empezaron a adquirir cierta autonomía. Por ejemplo, Lehtinen y Lehtinen (1982) señalan que los servicios son actividades intangibles en las que intervienen un proveedor y un cliente con el fin de crear la satisfacción de este último. Para Fisher y Navarro (1994) un servicio es un tipo de bien económico que constituye lo que se conoce como sector terciario (es decir, todo el que trabaja y no produce bienes se supone que produce servicios).

La palabra servicio manifiesta una particularidad del proceso, donde la actividad y el resultado coinciden en tiempo y espacio. El proceso de producción del servicio es, a su vez, el proceso de consumo de este. Es decir, lo que se produce es al mismo tiempo lo que se consume. Kotler (1997) manifiesta que un servicio es toda actividad que una parte ofrece a otra, y se caracteriza por ser intangible y por no dar lugar a la propiedad de ninguna cosa, pues su producción no está necesariamente vinculada a un producto físico. Para Schroeder (1992) un servicio nunca existe: solamente se puede observar el resultado despues del hecho. Por su parte, Colunga (1995) indica que el servicio es el trabajo que realizan otras personas. 


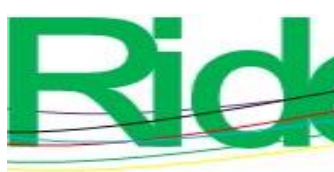

Revista Iberoamericana para la Investigación y el Desarrollo Educativo ISSN 2007 - 7467

En pocas palabras, se puede decir que el objetivo fundamental de un servicio es la satisfacción del cliente o usuario, lo que se verá reflejado generalmente en sus intenciones de volver a comprar o usar. Por eso, siempre se deben tomar en cuenta los intereses de los consumidores, de ahí que los formatos incomprensibles o complicados atenten contra el buen servicio.

\section{Calidad del servicio}

La globalización y los acelerados cambios tecnológicos han generado un mercado cambiante, caracterizado por la aparición de nuevos competidores. Puede señalarse que a partir los años 70 el mercado dejó de estar centrado en los vendedores para enfocarse en los compradores. La atención se enfocó en las preferencias del consumidor, lo que provocó el cambió en la mercadotecnia de un enfoque de fabricar y vender hacia un concepto de detectar y responder. Las empresas, por tanto, ahora examinan primero las preferencias de sus clientes para después reaccionar ofreciéndoles los productos y servicios que demandan.

Desde sus inicios, la calidad del servicio ha sido difícil de medir en comparación con los productos debido a sus características intangibles, subjetivas, inseparables, heterogéneas y de caducidad (Lovelock, 2011). Cronin y Taylor (1992) comentan que la calidad del servicio es lo que el cliente percibe del nivel de desempeño del servicio prestado. En el área de servicios, la calidad representa más que el cumplimiento de los estándares, pues estos se alcanzan con facilidad si las partes están comprometidas con las funciones que realizan. La calidad significa marcar una diferencia, impactar en la percepción del cliente y garantizar altos niveles de satisfacción y retorno (Rodríguez, s. f.).

La calidad del servicio y la satisfacción del cliente son conceptos que se encuentran interrelacionados, pues una mayor calidad del servicio incrementa la satisfacción del consumidor. En otras palabras, la calidad del servicio puede entenderse como todo aquello que es necesario hacer para lograr la satisfacción del consumidor. Por eso, un servicio puede ser percibido y valorado por los usuarios como malo, deficiente o insatisfactorio, o como exitoso y satisfactorio.

Oliver (1980) señala que un cliente satisfecho con el servicio está más dispuesto a utilizarlo de nuevo y a recomendarlo a otros. De hecho, mientras que los usuarios satisfechos transmiten sus experiencias positivas a un promedio de tres personas, los insatisfechos lo hacen a un promedio de trece personas (Kotler, 1997). Esto significa que existen hasta cuatro veces más probabilidades de crear una imagen negativa de un 


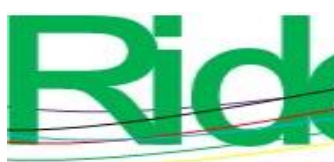

Revista Iberoamericana para la Investigación y el Desarrollo Educativo ISSN 2007 - 7467

servicio, por lo que un usuario satisfecho es el mejor recurso de promoción y la mejor maniobra de imagen (Hobohm, 2002).

La calidad es un concepto indefinible, lo que hace sumamente complejo el proceso de su medición, y los servicios son esencialmente intangibles, lo cual supone diseñar procedimientos que hagan tangible esa propiedad. Estudiar la satisfacción del servicio es importante porque este concepto constituye un indicador de calidad que influye en el estado de ánimo de los usuarios y tiene repercusiones en los niveles de productividad, ausentismo y costos. Por tanto, se debe contar con usuarios satisfechos con los servicios para alcanzar los objetivos de la institución.

\section{Satisfacción del usuario}

Gierl (1993) menciona que la satisfacción del usuario no es una condición para asegurar su conservación, aunque sí es necesaria. Según Simon y Homburg (1997), esta satisfacción es el resultado de un proceso constante de comparación entre la experiencia y la percepción subjetiva, por un lado, y las expectativas y los objetivos, por el otro. Los resultados pueden ser a) insatisfacción del usuario, b) satisfacción del usuario y c) entusiasmo del usuario.

En este sentido, se hace necesario que las expectativas de calidad de los usuarios se encuentren influenciadas por la experiencia previa que tengan con la institución y por las informaciones que les hayan transmitido otras personas. Asimismo, la imagen de la institución y el desarrollo de un modelo de identidad corporativa juegan un papel importante.

Hobohm (2002) manifiesta que un usuario que no se siente a gusto cuando utiliza los servicios tiene más probabilidades de divulgar una baja calidad, en comparación con un usuario que pueda soportar fallas tangibles siempre y cuando lo hayan atendido de forma servicial. Un nivel de calidad alto se logra cuando los usuarios están satisfechos o cuando superan sus expectativas. Al generarse entusiasmo entre los usuarios se fortalece su fidelidad y permite que se transmita una publicidad positiva de boca en boca.

En los años 90 quedó demostrado que la satisfacción del usuario tiene un efecto positivo, aunque la conservación del usuario es mucho más importante para el éxito (Reichheld y Sasser, 1998). En las instituciones públicas, el diálogo con los usuarios tiene mayor influencia en la conservación de ellos que otros elementos de satisfacción. Homburg, Giering y Hentschel (1999) manifiestan que la conservación del usuario está integrada por un comportamiento previo (compra y recomendaciones) y un comportamiento propuesto (intensiones de volver a consumir, de consumir más y de 


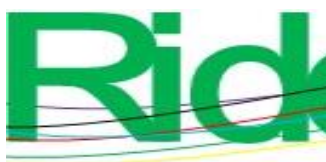

Revista Iberoamericana para la Investigación y el Desarrollo Educativo ISSN 2007 - 7467

recomendar). Asimismo, mientras que la fidelidad del usuario mantiene la perspectiva desde el punto de vista de la demanda, la conservación del usuario comprende tanto la oferta como la demanda.

Heinrichs y Klein (2001) señalan que la gestión de conservación del usuario se refiere a la sistematización del análisis, planeación, ejecución y control de las medidas enfocadas a mantener y cultivar una relación de intercambio con los usuarios de una institución. Meffert y Bruhn (2000) refieren que asentar la confianza desde una perspectiva planificada se considera como una condición necesaria para cualquier relación permanente, tanto para un interlocutor interno como uno externo. La relación entre proveedor y cliente se observa como un intercambio en el que todo el equipo se identifica con el principio de orientación del usuario y con las metas de la institución en la que labora.

\section{Principales modelos de calidad en los servicios}

La calidad en los servicios tienen que ser evaluados, pues no puede hablarse de evaluación si no es para la mejora de la calidad existente (Garbanzo-Vargas, 2014). Básicamente, se reconocen dos enfoques fundamentales como los más significativos en el estudio de la calidad del servicio: a) modelos basados en el paradigma de las discrepancias, que define la calidad del servicio como calidad percibida, resultado de la comparación o de la diferencia entre expectativas y percepciones, cuyo principal representante es el instrumento Servqual (Parasuraman, Zeithaml y Berry, 1985); y b) modelos basados en el paradigma de la percepción del desempeño, que define la calidad como percibida, pero resultado de la percepción del desempeño del servicio prestado, en el que destaca el instrumento Servperf (Cronin y Taylor, 1994).

Parasuraman et al. (1985) crearon el modelo y el instrumento de evaluación Servqual (service quality) con base en sus investigaciones en diferentes tipos de servicios. El modelo define la calidad del servicio como un desajuste entre las expectativas previas al consumo del servicio y la percepción del servicio prestado, determinando que la calidad puede ser medida mediante la diferencia entre ambos conceptos. Asimismo, el modelo propone que la calidad del servicio se puede medir mediante cinco dimensiones:

a) Elementos tangibles: Se refiere a la apariencia de las instalaciones físicas, equipo, personal y materiales para comunicaciones.

b) Confiabilidad: Capacidad para proporcionar el servicio prometido de manera precisa, amable y cuidadosa. 
c) Capacidad de respuesta: Consiste en la buena disposición y voluntad del personal para ayudar a los clientes y otorgarles un servicio ágil.

d) Seguridad: Es el conocimiento y cortesía de los trabajadores, así como su capacidad para inspirar seguridad y confianza.

e) Empatía: Se refiere al cuidado y atención individualizada que la organización proporciona a sus clientes.

Estas dimensiones se miden en una escala de dos secciones de 22 puntos cada una; la primera registra las expectativas de los clientes de empresas excelentes en la industria de los servicios, y la segunda mide la percepción de los consumidores de una empresa dada. Los resultados se comparan para determinar las calificaciones de las brechas de cada una de las cinco dimensiones. En general, se mide la diferencia entre el servicio esperado y el servicio percibido.

El modelo Servperf (Cronin y Taylor, 1992) surge debido a las críticas del modelo Servqual. Los autores señalan que el Servqual no es el más adecuado para medir la calidad del servicio debido a deficiencias encontradas. Por ejemplo, indican que las expectativas son el componente del instrumento Servqual que más controversias ha presentado por los problemas de interpretación que expone a los encuestados, suponen una redundancia dentro del instrumento de medición, ya que las percepciones están influenciadas por las expectativas y por su variabilidad en los diversos momentos de la prestación del servicio.

Rodríguez (s. f.) señala que Cronin y Taylor (1992) —basados en diversos cuestionarios - contrastaron la medición de la calidad realizada a través de la diferencia entre expectativas y percepciones de los consumidores y la realizada únicamente con las percepciones o actitudes de ellos. Estos investigadores utilizaron los 22 ítems del Serqual; por una lado, propusieron utilizar el Serqual y, por el otro, medir la calidad tomando en cuenta solo las percepciones con las mismas cinco dimensiones e ítems del Servqual. De este modo surgió el instrumento Servperf.

Medir la calidad del servicio con base en el desempeño - como lo hace el Servperf - reduce la amplitud del cuestionario, lo que permite una mayor colaboración del encuestado y una fácil comprensión, en vez de utilizar dos columnas que crean confusión y complejidad. Además, el Servperf requiere de menos tiempo para administrar el cuestionario, predice mejor la satisfacción, y el trabajo de interpretación y análisis es más fácil de realizar.

Rodríguez (s. f.) refiere que de acuerdo con la opinión de varios expertos — tales como Bolton y Drew (1991), Churchill y Suprenant (1982), Woofruff, Cadotte y Jenkins (1993), entre otros-, el instrumento Servperf parece conformar más de cerca las 


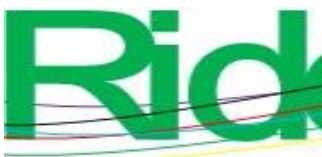

Revista Iberoamericana para la Investigación y el Desarrollo Educativo ISSN $2007-7467$

implicaciones sobre actitudes y satisfacción. Asimismo, la revisión de la literatura proporciona un soporte suficiente a la superioridad de la medida de la calidad del servicio basada únicamente en el desempeño. Por tanto, puede afirmarse que el Servperf tiene una mejor aceptación, ya que la medición exclusiva de la percepción del desempeño muestra con mayor exactitud la realidad del servicio prestado.

\section{Planteamiento del estudio, objetivo y justificación}

El concepto calidad fue inicialmente utilizado en el medio empresarial y luego fue extendido al entorno educativo. Cuando este constructo se aplica a este último se debe contemplar tanto su finalidad como su sentido y sus funciones, es decir, todo el sistema educativo en su conjunto (Sverdlick, 2012). Jiménez, Terriques y Robles (2011) consideran que en la valoración de la calidad de la educación, la satisfacción del estudiante es un factor importante y se refleja en la eficiencia y eficacia de los servicios académicos y administrativos que se ofrecen, tales como programas de estudio, relaciones interpersonales, intalaciones y equipamiento, entre otros.

Asimismo, Araiza, Zambrano y Ramírez (2016) señalan que ciertos estudios se han enfocado en la valoración global de los estudiantes en torno al conjunto de servicios que ofrecen las IES, tales como los de apoyo escolar, los culturales, los deportivos y los sociales. Bustos, Salazar y Bermúdez (2012) indican que se puede hablar de calidad en la educación cuando esta es capaz de maximizar los beneficios profesionales, sociales y económicos, así como la calidad de vida de los estudiantes.

En las últimas dos décadas las IES han incrementado su interés por la evaluación de la calidad enfocada a la mejora continua institucional (Abarca, Cáceres, Jiménez, Moraleda y Romero, 2013). Tumino y Poitevin (2013) explican que los estudiantes son la razón de ser de las instituciones educativas, por lo que es importante tomar en cuenta las demandas que hacen sobre la calidad de los servicios, con la finalidad de mantener el prestigio y la imagen institucional.

Explicado lo anterior, se puede decir que el presente estudio se realizó en el departamento de Servicios Escolares (SE) de una institución de educación superior (IES) ubicada en México. La IES cuenta con nueve facultades donde se imparten 25 licenciaturas, ocho maestrías, seis especialidades médicas y varios centros de investigación. El departamento de SE inició sus operaciones a partir del año 1988. Actualmente, tiene como misión planear, organizar, operar y controlar los servicios escolares que ofrece con calidad y calidez humana en sus etapas de admisión, permanencia y egreso. Su visión es ser reconocida por la calidad, transparencia y 
oportunidad con que brinda los servicios escolares, utilizando tecnología de vanguardia y personal capacitado y ético, comprometido y sensible a las necesidades de la comunidad de la IES.

El departamento de SE tiene como principales objetivos coordinar y vigilar que se realicen a tiempo y en forma los procesos de selección y admisión de aspirantes a ingresar a la IES, realizar oportunamente el registro de inscripciones y reinscripciones, coordinar el registro y actualización del historial académico de los alumnos, validar los comprobantes de estudios y certificados, y mantener actualizados, bajo resguardo y protegidos los documentos y expedientes que conforman el historial académico de los alumnos, entre otros objetivos.

El departamento de SE plantea dentro de su misión, visión y objetivos ofrecer servicios de calidad. Esta preocupación se debe a que lo contrario puede afectar el ambiente y los resultados institucionales, pues la calidad en la educación se relaciona con la estructura y la gestión institucional, así como con los métodos para transmitir conocimientos (Capalleras y Veciana, 2004; Salvador, 2005).

La IES en estudio obtuvo la certificación de calidad de los servicios de la Organización Internacional de Normalización (ISO, por sus siglas en inglés) a partir del año 2007, estatus que ha logrado mantener hasta el año 2019. Una vez adquirido el compromiso, el organismo certificador debe evaluar a la institución durante dos ciclos anuales de vigilancia para poder renovar y conservar su certificación, lo que constituye un problema debido a que - en caso de no mantener la certificación luego de ser evaluada nuevamente por los organismos externos - dejaría de percibir determinados recursos de apoyo con los que cuenta actualmente - p. ej., el Programa de Fortalecimiento a la Excelencia Educativa (Profexce)—. Esto perjudicaría la adquisición de bienes tangibles, la funcionalidad, los tiempos de respuesta, la satisfacción y la imagen institucional.

Por tal motivo, el Sistema de Gestión de Calidad (SGC) de la institución en estudio tiene la necesidad de vigilar la adecuada operatividad de los procesos existentes, para lo cual requiere de información constante con la finalidad de optimizar la calidad de los servicios de forma continua.

Por lo anterior, se decidió realizar la presente investigación, la cual tiene como objetivo medir la calidad de los servicios escolares ofrecidos desde la percepción de los estudiantes de una facultad de la IES en estudio. Asimismo, esta investigación proporcionará información útil para la planeación estratégica y la toma de decisiones dirigida a la mejora de las condiciones de trabajo, los comportamientos y el desempeño del personal que presta el servicio. 


\section{Método}

\section{Tipo de estudio}

Este estudio es exploratorio y descriptivo. Primero se realizó una medición cuantitativa y posteriormente se aplicaron entrevistas cualitativas con el propósito de profundizar en los resultados obtenidos. El estudio cualitativo se realizó mediante la técnica de grupos focales con ocho estudiantes, la cual permitió "reforzar teorías e identificar cuestiones críticas para investigaciones futuras" (Stake, 1994, p. 245). El diseño es no experimental con enfoque transversal, ya que no se tiene injerencia en los resultados (Hernández-Sampieri y Mendoza, 2018).

\section{Participantes}

Se consideró solo a los estudiantes de nuevo ingreso debido a que son los que más utilizan el servicio de atención al público, pues posteriormente son atendidos en línea. De un total 280 estudiantes de nuevo ingreso de una facultad de la IES en estudio, se calculó una muestra con $95 \%$ de confiabilidad y un error permitido de $5 \%$, con probabilidades $p$ y $q$ de 0.5 , respectivamente. Se obtuvo una muestra de 162 estudiantes, de los cuales 81 son de la licenciatura en Contaduría y 81 de la licenciatura en Administración y Finanzas.

\section{Instrumentos}

Para la recolección de datos cuantitativos se utilizó el instrumento Servperf de Cronin y Taylor (1994), basado en el modelo de calidad del servicio percibido (Servqual; (Parasuraman et al., 1985) y adaptado por Pérez y Ramírez (2016).

Este instrumento evalúa cinco dimensiones: elementos tangibles, confiabilidad, capacidad de respuesta, seguridad y empatía. Consta de 25 ítems con cinco alternativas de respuesta cada uno: 1) totalmente en desacuerdo, 2) en desacuerdo, 3) ni de acuerdo ni en desacuerdo, 4) de acuerdo, 5) totalmente de acuerdo. Pueden obtenerse puntajes mínimos de 25 y máximos de 125. La validez del instrumente se dio mediante la opinión de expertos y la prueba de Lawshe; los resultados de la muestra piloto fueron de 0.80 (esto demuestra que los ítems son aceptables para la medición). Asimismo, el análisis estadístico obtuvo un coeficiente de confiabilidad alfa de Cronbach de .949. Para la obtención de los datos cualitativos se elaboró una guía de entrevistas con preguntas semiestructurada basadas en los resultados obtenidos y las cinco dimensiones del cuestionario Servperf. 


\section{Procedimiento}

El análisis de los datos se realizó mediante el siguiente proceso:

a) Para la administración del instrumento Servperf se contó con la ayuda de estudiantes de la IES en estudio. Los cuestionarios se aplicaron en las aulas de la institución mediante previa cita. Se necesitaron cuatro sesiones para completar la recolección de datos. Con la información obtenida se creó una base de datos, los cuales se capturaron utilizando el paquete estadístico para las ciencias sociales SPSS (Statistic Package for Social Sciencie) versión 17.0 para Windows.

b) Se analizaron los datos obtenidos utilizando estadística descriptiva, tomando como medida de tendencia central la media y como medida de dispersión la desviación estándar.

c) Se elaboró una guía de entrevistas semiestructuradas para profundizar sobre los resultados mediante la técnica de grupos focales.

d) Se creó un clima de confianza que contribuyó a la sinceridad de las respuestas.

e) La recolección de datos generó múltiples enunciados, testimonios, documentos, reportes de investigación y resumenes de datos.

f) La información se organizó mediante categorías y tablas de frecuencias permitiendo identificar y analizar el objetivo del estudio.

\section{Resultados}

\section{Medición cuantitativa}

La tabla 1 muestra los resultados de la administración del instrumento Servperf a los estudiantes.

Tabla 1. Calidad del servicio ofrecido desde la perspectiva de los alumnos

\begin{tabular}{|l|c|c|c|}
\hline Dimensiones & $\mathrm{N}$ & Media & $\begin{array}{c}\text { Desviación } \\
\text { estándar }\end{array}$ \\
\hline Elementos tangibles & 162 & 4.12 & .97 \\
\hline Confiabilidad & 162 & 3.96 & .95 \\
\hline $\begin{array}{l}\text { Capacidad de } \\
\text { respuesta }\end{array}$ & 162 & 3.48 & .04 \\
\hline Seguridad & 162 & 3.98 & .01 \\
\hline $\begin{array}{l}\text { Empatía } \\
\text { Servicio General }\end{array}$ & 162 & 3.49 & .02 \\
\hline
\end{tabular}

Fuente: Elaboración propia 


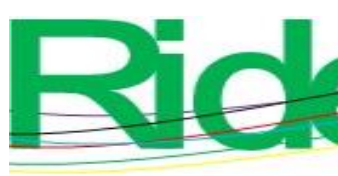

Revista lberoamericana para la Investigación y el Desarrollo Educativo ISSN $2007-7467$

En la tabla 1 se muestra que los estudiantes están de acuerdo con los elementos tangibles (4.12), con la confiabilidad (3.96) y con la seguridad (3.98) que ofrece el departamento de SE. La certificación de los servicios escolares con que cuenta la institución les ha permitido contar con ciertos recusos de apoyo que han utilizado para la mejora de la apariencia de sus instalaciones, equipo y personal. Asimismo, debido a que el personal ha recibido diversos cursos de capacitación técnica, cometen un mínimo de errores, lo que contribuye a la solución de problemas, y genera cierta seguridad y confianza en los estudiantes.

Además, los participantes no están de acuerdo ni en desacuerdo con la capacidad de respuesta (3.48) y con la empatía (3.49) que proporciona el departamento de SE. La institución cuenta con 7375 estudiantes matriculados, por lo que en periodos de inscripciones el servicio se satura y es frecuente encontrar estudiantes descontentos o molestos con el trato y los tiempos de respuestas. En tal sentido, la calidad del servicio debe mostrar empatía en todo momento, ponerse en el lugar del estudiante y averiguar realmente lo que quiere, por encima del enojo o las protestas.

En cuanto a la calidad del servicio en general que presta el departamento de SE, los estudiantes están de acuerdo (3.80), pues perciben que es apropiada debido, principalmente, a que utilizan las tecnologías de información en sus procesos, acorde a los tiempos modernos. Cabe señalar que la certificación de la que goza la institución ha traído beneficios para toda la comunidad de la IES en estudio.

\section{Evaluación cualitativa}

Cuando se entrevistó a los estudiantes sobre los elementos tangibles (4.12), señalaron que el departamento de SE cuenta con equipo de cómputo funcional para la rápida solución de peticiones y que la mayoría de las veces el personal apoya en la solución de problemas a los estudiantes. En cuanto a los uniformes comentaron que todos cumplen con la norma. Un estudiante comentó: "Van presentables y la apariencia física es adecuada".

Al preguntar a los estudiantes sobre la confiabilidad (3.96), señalaron que "al inicio de cada semestre el servicio es tardado, pero se entiende porque son demasiados los estudiantes y el número de computadoras es limitado". Otro estudiante demandó que deberían agilizarse los tiempos de respuesta sobre los trámites solicitados, pues a veces se retrasan de dos a tres días. Los estudiantes señalaron que no utilizan el buzón de quejas y sugerencias; incluso hubo quien señaló que "no tenía conocimiento de su existencia". 


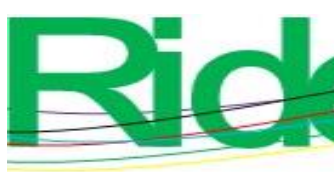

Revista Iberoamericana para la Investigación y el Desarrollo Educativo ISSN $2007-7467$

Cuando se cuestionó a los entrevistados sobre la capacidad de respuesta (3.48), uno de ellos manifestó que en ocasiones se demoran los trámites. Al respecto se tiene el siguiente testimonio: “A veces, los documentos no están listos y hay que esperar". Mencionaron que esto les incomoda, ya que piden permiso dentro de su horario de clases para realizar sus trámites.

Sobre la seguridad (3.98), los estudiantes manifestaron que la mayoría del personal los tratan con amabilidad, son positivos para atenderlos y muestran interés en ayudarlos, pero no todos son así. Asimismo, comentaron que el personal se encuentra capacitado para atenderlos, pero que en ocasiones no lo hacen. Un entrevistado dijo: “A pesar del tiempo que demoran para cumplir con un trámite, siempre lo realizan correctamente".

En relación con la empatía (3.49), los estudiantes comentaron que el personal no siempre muestra una actitud positiva, amable e interesada para atender a quien lo solicita. Al respecto, un estudiante señaló: "Me han tocado personas amables que aclaran mis dudas, pero de igual forma hay personas que no atienden como se debiera".

En cuanto a la evaluación general (3.80) del servicio que ofrece el departamento de SE, los estudiantes comentaron que estan de acuerdo. Sin embargo, se observan algunos problemas relacionados con el trato que reciben los estudiantes al ser atendidos por ciertos empleados. Un estudiante señaló que los empleados deben saber controlar sus emociones. Al respecto, se tiene el siguiente testimonio: "Nosotros no tenemos la culpa de lo que les pasa, a veces están alterados y deberían darles un tiempo para que se relajen”.

\section{Discusión}

Medir la calidad de los servicios escolares significa para el Sistema de Gestión de Calidad de la institución contar con indicadores de control sobre dichos servicios, lo cual permite solventar las irregularidades detectadas de manera anticipada y mantener la certificación de la IES.

En este estudio se encontró que para los estudiantes la calidad de los servicios escolares en general es apropiada. Sin embargo, vale acotar que solo en tres de las cinco dimensiones que integran dicho constructo (elementos tangibles, confiabilidad y seguridad) se tiene una calidad aceptable, según la percepción de los estudiantes. Estos resultados son contrarios con lo encontrado por Bayraktaroglu y Atrek (2017), quienes hallaron una menor relevancia de los elementos tangibles y una alta interacción personal en el sector de la educación. Esto significa que los servicios ofrecidos, en este caso, 


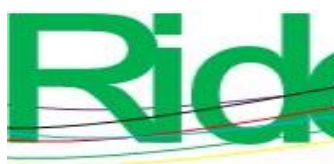

Revista Iberoamericana para la Investigación y el Desarrollo Educativo ISSN 2007 - 7467

utilizan muy poca tecnología en sus procesos y existe buena comunicación entre la comunidad educativa de la institución, lo que constituyen factores importantes para tomar en cuenta en la mejora de la calidad de los servicios.

Asimismo, en dos de las cinco dimensiones que integran el concepto de calidad se requiere atención especial antes de que la situación empeore y ponga en riesgo la certificación de la calidad con la que se cuenta. Estas dos dimensiones están ocasionando que los estudiantes sientan cierto descontento, por lo que se sugiere crear las estrategias adecuadas para actuar con empatía y corregir la capacidad de respuesta. Esto es consistente con lo reportado por Sánchez, Navas, Chávez, Miranda y Morocho (2019), quienes han señalado que la empatía es el principal problema derivado de la falta de capacitación del talento humano. En este sentido, en el presente trabajo se ha detectado que la capacidad para ponerse en el lugar del estudiante está generando problemas, por lo que no basta con capacitarse en las áreas báscas para desempeñar bien el trabajo, sino que hace falta conocer y practicar las técnicas para mejorar las relaciones humanas.

Diversos estudios han sido consistentes al medir la calidad del servicio en general. No obstante, pueden observarse variaciones en la medida de sus dimensiones, como sucedió en el presente estudio al obtener una calidad apropiada y ser congruente con los estudios realizados por Ibarra y Casas (2015), quienes encontraron una calidad aceptable en la medición de los servicios ofrecidos. Sin embargo, estos autores obtuvieron una calificación menor para la dimensión confiabilidad, mientras que el presente estudio obtuvo calificaciones menores para las dimensiones capacidad de respuesta y empatía.

Por otra parte, la fortaleza del presente estudio radica en que los resultados de la medición cuantitativa de la calidad fueron reforzados mediante la administración de entrevistas de corte cualitativo, lo que permitió conocer a profundidad y detalle los motivos de las aprobaciones y los descontentos de los estudiantes en relación con la calidad del servicio ofrecido, pues los números solo proveen información limitada para el análisis. Asimismo, el estudio contribuye a la literatura de investigación acerca de la calidad en los servicios escolares en las IES. Finalmente, dentro de las limitaciones se tiene que el estudio solo se enfocó en las percepciones de los estudiantes de una facultad en una IES. 


\section{Conclusiones}

En términos generales, los estudiantes perciben que el servicio que ofrece el departamento de SE es de adecuada calidad. Las calificaciones más bajas se presentan en las dimensiones capacidad de respuesta y empatía debido a que prevalece como principal inconveniente la actitud que asume una parte del personal del departamento de SE, caracterizada - en ocasiones - por la falta de amabilidad, disposición y tiempo de respuesta. Se requiere, por tanto, que los administradores inspiren en estos empleados el sentido de importancia y el significado que tiene el servicio a los estudiantes, plasmados en su misión, visión y objetivos, pues pareciera que les falta una mayor identificación con la calidad del servicio que deben prestar.

En consecuencia, se propone que la institución fomente una cultura de sensibilización del servicio, mediante la implementación de un programa de capacitación permanente de atención a los estudiantes, que sea congruente con los objetivos institucionales. Además, se sugiere que dicho programa se sustente en la teoría del comportamiento organizacional, en donde todos los trabajadores practiquen la empatía en situaciones difíciles, sepan escuchar, sean amigables, corteses, accesibles y expertos. Asimismo, estén listos para responder a las necesidades de los estudiantes con las palabras y tono adecuados, y estar dispuestos a hacer lo que sea necesario para agradarles. Esto es consistente con Robbins y Judge (2017), quienes mencionan que el comportamiento organizacional ofrece una guía valiosa para ayudar a que las instituciones creen esa cultura.

Por otra parte, se recomienda establecer un sistema de evaluación constante en la institución, en donde el modelo Servperf contribuya como una herramienta de apoyo eficaz para medir y analizar propuestas de mejora de la calidad en la prestación de los servicios escolares. Igualmente, se recomienda tomar en cuenta las opiniones, quejas, sugerencias y expectativas de los estudiantes para determinar los niveles de satisfacción y establecer medidas correctivas en casos de ineficiencia operativa o críticas negativas. De esta manera, se contará con información relevante de los usuarios para una adecuada toma de decisiones sobre las actividades que deberán desarrollarse para lograr la mejora continua de la calidad de los servicios escolares ofrecidos. 


\section{Futuras líneas de Investigación}

Se hace necesario, en definitiva, realizar mayor investigación para consolidar los resultados obtenidos, así como estudios de caso y etnográficos. Futuras investigaciones podrían medir la calidad de los servicios escolares desde la percepción de los administradores escolares en otras facultades o en otras IES tanto públicas como privadas, para establecer comparaciones con los presentes resultados. Asimismo, futuras líneas de investigación podrían realizar estudios para conocer si existe relación entre la calidad de los servicios escolares y otros constructos, tales como la satisfacción, el clima o la cultura organizacional.

\section{Referencias}

Abarca, F., Cáceres, S., Jiménez, E., Moraleda, V. y Romero, B. (2013). Satisfacción de los alumnos con la institución universitaria y el rendimiento académico. Reidocrea, Revista Electrónica de Investigación Docencia Creativa, 2, 48-53. Recuperado de https://digibug.ugr.es/handle/10481/27613

Araiza, V. M. J., Zambrano, E. A., y Ramírez, R., J. F. (2016). Calidad de los servicios universitarios: una percepción desde sus actores en una escuela de negocios. Innovaciones de Negocios, 13(25), 071-095.

Bayraktaroglu, G. y Atrek, B. (2017). Prueba de superioridad y dimensionalidad de SERVQLAL frente a SEVPERF en educación superior. Taylor \& Francis Online, 17(1), 47-59, Doi: 10.1080/10686967.2010.11918260

Bustos, T. C., Salazar, A. M. y Bermúndez, J. J. (2012). Percepción de directivos, docentes y estudiantes, en torno a la implementación de un sistema de aseguramiento de la calidad en las instituciones educativas (tesis de licenciatura). Chía: Universidad de La Sabana.

Capalleras, J. L. y Veciana, J. M. (2004). Calidad de servicio en la enseñanza universitaria: desarrollo y validación de una escala de medida. Revista Europea de Dirección y Economía de la Empresa, 13(4), 55-72.

Colunga, C. (1995). La calidad en el servicio. México: Panorama Editorial.

Cronin, J. and Taylor, S. (1992). Measuring service quality: a reexamination and extension. Journal of Marketing, 56(3), 55-68.

Cronin, J. and Taylor, S. (1994). SERVPERF versus SERVQUAL: reconciling performance based and perceptions minus expectations measurement of service quality. Journal of Marketing, 58(19), 125-131. 


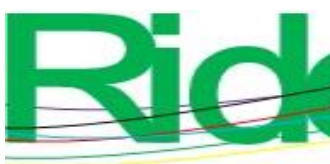

Revista Iberoamericana para la Investigación y el Desarrollo Educativo ISSN $2007-7467$

Fisher, I. y Navarro, V. (1994). Introducción a la investigación de mercado (3. ${ }^{\mathrm{a}}$ ed.). México: McGraw-Hill Interamericana.

Garbanzo-Vargas, G. M. (2014). Calidad y equidad de la educación superior pública. Aspectos por considerar en su interpretación. Revista Educación, 31(2), 11-27.

Gierl, H. (1993). Zufriedene Kunden als Markenwechsler. Absatzwirtschaft, 36(2), 9094.

Heinrichs, W. y Klein, A. (2001). Kulturmanagement von A-Z. 600 Begriffe für Studium un Praxis (2. ${ }^{\mathrm{a}}$ ed.). Beck-Wirtschaftsberater im dtv. 5877, Munich.

Hernández-Sampieri, R. y Mendoza, C. (2018). Metodología de la investigación. Las rutas cuantitativa, cualitativa y mixta (1. ${ }^{\mathrm{a}}$ ed.). México: McGraw Hill.

Hobohm, H. (2002). Kundenbindung und Qualitätsmanagement. In Hobohm/Umlauf, Handmarke 3/5.

Homburg, Ch., Giering, A. and Hentschel, F. (1999). Der Zusammenhang zwischen Kundenzufriedenheit und Kundenbindung. En Bruhn/Homburg. pp. 81-112.

Ibarra, L. y Casas, E. (2015). Aplicación del modelo Servperf en los centros de atención Telcel, Hermosillo: una medición de la calidad en el servicio. Contaduría y Administración, 60(1), 229-260.

Jiménez, A., Terriquez, B. y Robles, F. (2011). Evaluación de la satisfacción académica de los estudiantes de la Universidad Autónoma de Nayarit. Revista Fuente, 3(6), 46-56. Recuperado de http://fuente.uan.edu.mx/publicaciones/02-06/8.pdf

Kotler, P. (1997). Mercadotecnia. México: Prentice Hall.

Lehtinen, Jr. and Lehtinen, O. (1982). Service quality: a study of quality dimensions. Unpublished working paper, Helsinki: Service Management Institute.

Lovelock, C. (2011). Services marketing: People, technology, strategy. Harlow: Pearson Education.

Meffert, H. y Bruhn, M. (2000). Dienstleistungsmarketing. Grundlagen, Konzepte, Methoden (3. ${ }^{\mathrm{a}}$ ed.). Wiesbaden.

Oliver, R. (1980). A congnitive model of the antecedents and consequences of satisfaction decision. Journal of Marketing Research, 17(4), 460-469.

Parasuraman, A., Zeithaml, V. and Berry, L. (1985). A conceptual model of service quality and its implications for future research. Journal of Marketing, 49(85), 4150.

Pérez, D. y Ramírez, M. (2016). Diagnóstico de satisfacción de los usuarios del departamento de servicios escolares de la Escuela Superior de Agricultura del Valle del Fuerte. Revista Global de Negocios, 4(7), 13-25. 


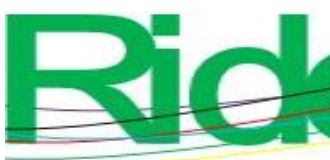

Revista Iberoamericana para la Investigación y el Desarrollo Educativo ISSN 2007 - 7467

Reichheld, F. and Sasser, E. (1998). Zero-Migration. Dienstleister im sog der qualitätsrevolution. Harvard Manager, 4, 108-116.

Robbins, S. y Judge, T. (2017). Comportamiento organizacional (17. ${ }^{\mathrm{a}}$ ed.). México: Pearson Educación.

Rodríguez, A. (s. f.). Elaboración del marco teórico referencial que sustente la aplicación de la metodología SERVPERF para la gestión de la calidad en una empresa de servicios. GestioPolis. Recuperado de https://www.gestiopolis.com/modeloservperf-para-gestion-calidad-empresa-servicios-marco-teorico/

Salvador, C. M. (2005). La percepción del cliente de los elementos determinantes de la calidad del servicio universitario: características del servicio y habilidades profesionales. Papeles del Psicólogo, 26(90), 1-9.

Sánchez, L. J. C., Nava, Z. S. A., Chávez, R. J. I., Miranda, M. F., y Morocho Q. J. C. (2019). Implementación del modelo Servperf como herramienta para el diagnóstico y propuesta de mejoras en la prestación del servicio. Caso: Instituto Ecuatoriano de Seguridad Social Agencia Riobamba. Ciencia Digital, 3(2), 475483. Doi: https://doi.org/10.33262/cienciadigital.v3i2.445

Schroeder, R. G. (1992). Administración de operaciones (3. ${ }^{\mathrm{a}}$ ed.). México: McGraw Hill Interamericana.

Simon, H. and Homburg, Ch. (1997). Kundenzufriedenheit (2. ${ }^{\mathrm{a}}$ ed.). Wiesbaden.

Stake, R. (1994). Case studies. In Norman, K. (1994), Handbook of Qualitative Inquiry (pp. 236-247). Thousand Oaks: CA.

Sverdlick, I. (2012). ¿Qué hay de nuevo en evaluación educativa? Buenos Aires: Centro de Publicaciones Educativas y Material Didáctico.

Tumino, M. C. y Poitevin, E. R. (2013). Evaluación de la calidad de servicio universitario desde la percepción de estudiantes y docentes: caso de estudio. REICE. Revista Iberoamericana sobre Calidad, Eficacia y Cambio en la Educación, 12(2), 63-84. Recuperado de https://dialnet.unirioja.es/descarga/articulo/4679055.pdf 